\title{
PRODUÇÃO DE ALFACE-AMERICANA, EM FUNÇÃO DE DOSES E ÉPOCAS DE APLICAÇÃO DE ZINÇO
}

\author{
Iceberg lettuce production in function of doses and application time of zinc \\ Jony Eishi Yuri', Geraldo Milanez de Resende² ${ }^{2}$ José Hortêncio Mota ${ }^{3}$, \\ Rovilson José de Souza ${ }^{4}$, Janice Guedes de Carvalho ${ }^{5}$
}

\begin{abstract}
RESUMO
Com o objetivo de avaliar a influência de doses de sulfato de zinco sobre a da alface- americana (Lactuca sativa L.), foram conduzidos três experimentos distintos entre os meses de maio e julho de 2002, no município de Três Pontas - MG. Utilizou-se o delineamento de blocos ao acaso, com cinco doses de sulfato de zinco $\left(0 ; 0,9 ; 1,8 ; 2,7\right.$ e $\left.3,6 \mathrm{~kg} \mathrm{ha}^{-1}\right)$ e 4 repetições, aplicadas em três diferentes épocas via foliar (14, 21 e 28 dias após o transplante). Foram avaliadas as características massa fresca total e comercial $\left(\mathrm{g}\right.$ planta $\left.^{-1}\right)$, circunferência da cabeça comercial $(\mathrm{cm})$ e comprimento do caule da cabeça comercial $(\mathrm{cm})$. As características massa fresca total e comercial evidenciaram efeitos significativos independentes para as doses de sulfato de zinco e épocas de aplicação Para massa fresca total, a dose de $2,01 \mathrm{~kg} \mathrm{ha}^{-1}$ de sulfato de zinco proporcionou o maior rendimento. Em relação à época de aplicação, essa característica apresentou o maior rendimento $\left(988 \mathrm{~g} \mathrm{planta}^{-1}\right)$ quando a pulverização foi realizada aos 14 dias. Em termos de massa fresca comercial, a dose de 2,09 $\mathrm{kg} \mathrm{ha}^{-1}$ propiciou o maior rendimento, sendo a aplicação aos 14 dias, estatisticamente superiores, com rendimentos de $593 \mathrm{~g} \mathrm{planta}^{-1}$. Para circunferência de cabeça, a dose de $2,63 \mathrm{~kg} \mathrm{ha}^{-1}$ de sulfato de zinco proporcionou o maior retorno. Não se observou efeitos significativos dos tratamentos para comprimento do caule.
\end{abstract}

Termos para indexação: Lactuca sativa L., nutrição de plantas, micronutriente.

\begin{abstract}
With the objective of evaluating the influence of doses of zinc sulphate on yield and quality of iceberg lettuce (Lactuca sativa L.), three distinct trials were carried out at Três Pontas, State of Minas Gerais, Brazil, from May to June of 2002 . The experimental design was randomized blocks with five doses of zinc sulphate $\left(0,0.9,1.8,2.7\right.$ and $\left.3.6 \mathrm{~kg} \mathrm{ha}^{-1}\right)$, applied to the foliage at three different application times (14, 21 and 28 days after transplanting), and four replicates. The characteristics total and commercial fresh mass $\left(\mathrm{g}_{\text {plant }}{ }^{-1}\right)$, commercial head circumference $(\mathrm{cm})$ and stem length of commercial head $(\mathrm{cm})$ were evaluated. The total and commercial fresh mass characteristics evidenced independent significative effects to the doses of zinc sulphate and application times. For total fresh mass, the dose of $2.01 \mathrm{~kg} \mathrm{ha}^{-1}$ of zinc sulphate was estimated to present the highest yield. In relation to application time, this characteristic was estimated to present the highest yield $\left(988 \mathrm{~g} \mathrm{plant}^{-1}\right)$ when the pulverization was realized at 14 days. The dose of $2.09 \mathrm{~kg} \mathrm{ha}^{-1}$ was estimated to achieve the highest yield for commercial fresh mass, the application at 14 days being statistically superior, with a yield of $593 \mathrm{~g}$ plant $^{-1}$. For head circumference, the dose of $2.63 \mathrm{~kg} \mathrm{ha}^{-1}$ of zinc sulphate was estimated to bring showed the highest return. No significant effects were observed for stem length.
\end{abstract}

Index terms: Lactuca sativa L., plant nutrition, micronutrient.

(Recebido para publicação em 3 de outubro de 2003 e aprovado em 6 de setembro de 2005)

\section{INTRODUÇÃO}

A alface é a hortaliça folhosa mais consumida no País (SANTOS et al., 2001), sendo que a alface-americana vem adquirindo importância crescente. O plantio deste tipo de alface visa atender as redes de lanchonetes e, atualmente, tem-se constatado o aumento no consumo desta hortaliça também na forma de salada. De acordo com CEAGESP (2001), no ano de 2001, entre os diferentes tipos de alface comercializados, 29,6\% foram representados pela alface-americana.
Os solos brasileiros, de maneira geral, são pobres em zinco devido ao material de origem, uma vez que este elemento tem como fonte primária os minerais ferromagnesianos (CHESWORTH, 1991), que estão relacionados com rochas básicas, e a maioria dos solos do Brasil, são formados a partir de rochas ácidas e, ou, sedimentos. A produção em áreas de cerrado, normalmente pobre em micronutrientes, principalmente zinco e boro, torna a prática da adubação com esses nutrientes de grande importância para a cultura.

\footnotetext{
${ }^{1}$ Doutorando em Fitotecnia - Universidade Federal de Lavras/UFLA - Departamento de Agricultura/DAG - Cx. P. 3037 - $37.200-000$ - Lavras, MG jonyyuri@uol.com.br

${ }^{2}$ Pesquisador da EMBRAPA Semi-Árido - Cx. P. 23 - 56.300-000 - Petrolina, PE.

${ }^{3}$ Doutor, Professor da Universidade Luterana do Brasil/ULBRA.

${ }^{4}$ Doutor, Professor Titular de Olericultura - Universidade Federal de Lavras /UFLA - Departamento de Agricultura /DAG - Cx. P. 3037 - 37.200-000 Lavras, MG.

${ }^{5}$ Doutora, Professora Titular de Nutrição Mineral de Plantas - Universidade Federal de Lavras /UFLA - Departamento de Ciência do Solo/DCS Cx. P. 3037 - 37.200-000 - Lavras, MG.
} 
O zinco nas folhas está associado aos complexos de baixo peso molecular, íons livres e formas insolúveis na parede celular, podendo tornar-se inativo dentro da célula pela complexação com o fósforo. Sua essencialidade para a função e, ou, estrutura, de diversas desidrogenases, proteinases e peptidases encontradas em plantas foram relatadas por Welch \& Norvell (1993), sendo classificado como elemento parcialmente móvel na planta (PEASLEE et al., 1981). Este nutriente tem como função principal ser componente e ativador enzimático, estando diretamente envolvido no metabolismo do nitrogênio (FAQUIN, 1994). O zinco é importante para o crescimento (GREWAL et al., 1997) e para manutenção da integridade da membrana plasmática da raiz (CAKMAK \& MARSCHNER, 1988).

Os altos coeficientes de correlação encontrados por Kalyanaraman \& Sivagurunathan (1993), sugerem que a concentração de zinco e cobre nos tecidos é linearmente relacionado com a sua aplicação no solo. Em ambiente protegido Gomes et al. (1999), recomendam acrescentar na adubação de plantio da alface, $3,0 \mathrm{~kg} \mathrm{ha}^{-1}$ de zinco.

O efeito positivo da adição de zinco em alface é relatado por Moreira et al. (2001) que observaram com relação à matéria seca da planta inteira, no nível baixo de $P$, não haver efeito da adição de $\mathrm{Zn}$ na solução; sendo que no nível normal de $\mathrm{P}$, a adição de $\mathrm{Zn}$ proporcionou maior produção de matéria seca, e no nível alto de $\mathrm{P}$, os níveis normal e alto de $\mathrm{Zn}$ proporcionaram maiores incrementos na produção de matéria seca. Fontes et al. (1982) observaram um decréscimo médio de 25,5; 31,1 e 17,4\% em dois anos de estudo, quando na ausência da adubação com zinco, sobre a produção total e comercial e o peso médio da cabeça. Ao contrário, não foram encontrados efeitos do $\mathrm{Zn}$ e do $\mathrm{P}$ sobre área foliar e a produção de matéria seca da parte aérea e da raiz de alface, em meio hidropônico, de acordo com Iorio et al. (1996).

As informações relativas à aplicação de zinco na produção de alface, ainda são restritas e inconclusivas. Por essa razão, os produtores realizam pulverizações foliares semanais com produtos contendo zinco, sem a garantia de que este procedimento seja adequado, gerando preocupação quanto à sua eficiência e aproveitamento pela cultura.

Em razão do exposto, realizou-se um estudo com o objetivo de avaliar diferentes doses e épocas de aplicação de zinco via foliar no cultivo de alface-americana em condições de campo.

\section{MATERIALE MÉTODOS}

Foram realizados três experimentos em condições de campo, no período de fevereiro a abril de 2002, no município de Três Pontas, MG, Sul de Minas Gerais, situada a $21^{\circ} 22^{\prime} 00^{\prime \prime}$ de latitude Sul e $45^{\circ} 30^{\prime} 45^{\prime \prime}$ de longitude Oeste e a uma altitude de $870 \mathrm{~m}$ (IBGE, 2002). O solo da área experimental foi classificado como Latossolo Vermelho Distroférrico de textura argilosa (EMBRAPA, 1999). A análise do solo onde foi instalado o experimento apresentou as seguintes características químicas: $\mathrm{K}=48,0 \mathrm{mg} \mathrm{dm}^{-3} ; \mathrm{P}$ $=10,8 \mathrm{mg} \mathrm{dm}^{-3} ; \mathrm{Ca}=2,1 \mathrm{cmol} \mathrm{dm}_{c}^{-3} ; \mathrm{Mg}=0,8 \mathrm{cmol}_{\mathrm{c}} \mathrm{dm}^{-3}$; $\mathrm{Zn}=1,2 \mathrm{mgdm}^{-3} ; \mathrm{Fe}=29,8 \mathrm{mgdm}^{-3} ; \mathrm{Mn}=13,0 \mathrm{mgdm}^{-3} ; \mathrm{Cu}$ $=2,1 \mathrm{mg} \mathrm{dm}^{-3} ; \mathrm{B}=0,6 \mathrm{mg} \mathrm{dm}^{-3} ; \mathrm{pH} \mathrm{em} \mathrm{H}_{2} 0=5,8$ e matéria orgânica $=2,0$ dag $\mathrm{kg}^{-1}$.

Os três experimentos foram representados por três diferentes épocas de aplicação de sulfato de zinco (20\% de Zn), na cultura (14, 21 e 28 dias após o transplante). Em cada experimento, foram avaliados cinco doses de zinco $\left(0 ; 0,9 ; 1,8 ; 2,7\right.$ e $\left.3,6 \mathrm{~kg} \mathrm{ha}^{-1}\right)$, usando como fonte sulfato de zinco (20\% de $\mathrm{Zn}$ ). Os tratamentos foram dispostos no delineamento de blocos ao acaso com quatro repetições. As aplicações de sulfato de zinco foram realizadas por via foliar, por meio de pulverizador manual com $4 \mathrm{~L}$ de capacidade em máxima pressão, gastando-se $300 \mathrm{~L}$ de calda por hectare. Para evitar a ocorrência de deriva, no momento da aplicação, foi instalada ao redor da parcela uma cortina plástica com $1 \mathrm{~m}$ de altura.

As parcelas experimentais constituíram-se de canteiros com quatro linhas de 2,10 m de comprimento espaçadas de $0,30 \mathrm{~m}$, sendo entre plantas de $0,35 \mathrm{~m}$. As linhas centrais formaram a área útil, retirando-se duas plantas em cada extremidade. Para a adubação de plantio utilizou-se $1700 \mathrm{~kg} \mathrm{ha}^{-1}$ de formulado 04-14-08 e $1000 \mathrm{~kg} \mathrm{ha}^{-1} \mathrm{de}$ superfosfato simples. As adubações de cobertura foram realizadas por meio de fertirrigações diárias, totalizando 40 $\mathrm{kg} \mathrm{ha}^{-1}$ de $\mathrm{N}$ e $85 \mathrm{~kg} \mathrm{ha}^{-1}$ de $\mathrm{K}$, utilizando como fontes uréia e cloreto de potássio. O transplante das mudas foi feito em 22/05/2002, quando as mudas estavam com 31 dias de idade, irrigando-se diariamente, sendo a cultura conduzida sob "mulching" e os demais tratos culturais os comuns à cultura.

As colheitas foram realizadas em 23/07/2002 sendo avaliadas: a massa fresca total e comercial $\left(\mathrm{g} \mathrm{planta}^{-1}\right)$; circunferência da cabeça comercial $(\mathrm{cm})$ e comprimento do caule da cabeça comercial $(\mathrm{cm})$. Os dados foram submetidos à análise de variância conjunta e, observada a significância, procedeu-se ao teste de Tukey para épocas de aplicação e regressão polinomial para doses, com base no modelo quadrático, ao nível de 5\% de probabilidade.

Ciênc. agrotec., Lavras, v. 30, n. 4, p. 665-669, jul./ago., 2006 


\section{RESULTADOS E DISCUSSÃO}

Os resultados evidenciaram efeitos significativos independentes para as doses de sulfato de zinco e épocas de aplicação. Para massa fresca total, pela derivação da equação de regressão para o fator doses, constata-se que a aplicação de 2,01 $\mathrm{kg} \mathrm{ha}^{-1}$ de sulfato de zinco proporcionou o maior rendimento (Figura 1). Em relação à época, a aplicação aos 14 apresentou resultado estatisticamente superior, com rendimento de $988 \mathrm{~g}$ planta $^{-1}$ (Tabela 1).

Resultados similares foram observados para massa fresca comercial, em que se constatou que a dose de 2,09 $\mathrm{kg} \mathrm{ha}^{-1}$ propiciou o maior rendimento (Figura 1). Quanto à época de aplicação, aos 14, com $593 \mathrm{~g} \mathrm{planta}^{-1}$ de rendimento, foi estatisticamente superior (Tabela 1 ). $\mathrm{O}$ efeito positivo da adição de zinco em alface é relatado por Moreira et al. (2001) que observaram com relação à matéria seca da planta inteira, no nível normal de $\mathrm{P}$, que a adição de $\mathrm{Zn}$ proporcionou maior produção de matéria seca, e no nível alto de $\mathrm{P}$, os níveis normal e alto de $\mathrm{Zn}$ proporcionaram maiores incrementos na produção de matéria seca. Zinc (1966) não verificou efeito das aplicações de sulfato de zinco e de Zn-EDTA quando aplicados via foliar na cultura da alface. Contudo, as adubações de solo, com 11,2 a 38,1 $\mathrm{kg} \mathrm{ha}^{-1}$ de $\mathrm{Zn}$, foram muito favoráveis. A maior quantidade aplicada apresentou um efeito residual no solo de no mínimo quatro anos para a cultura.

Para circunferência de cabeça (Figura 2), observou-se apenas efeito para doses de sulfato de zinco, na qual a dose de $2,63 \mathrm{~kg} \mathrm{ha}^{-1}$ proporcionou o maior retorno. $\mathrm{O}$ comprimento do caule não apresentou diferenças estatísticas entre tratamentos. Esta é uma característica muito importante na cultura da alfaceamericana, principalmente, quando destinada à indústria de beneficiamento, devendo ser bastante reduzido, proporcionando menores perdas durante o processamento. Caule excessivamente comprido não apresenta boa compacidade e dificulta o beneficiamento, afetando a qualidade final do produto (YURI et al., 2002).

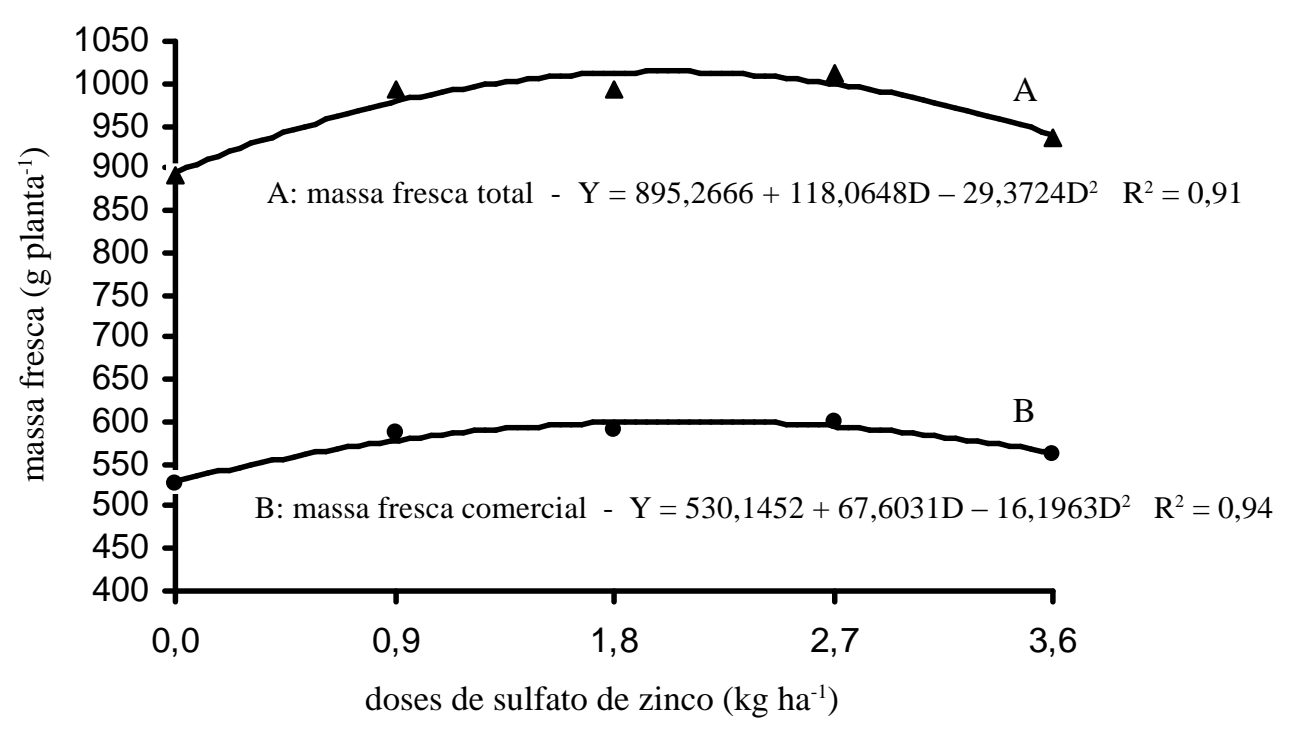

FIGURA 1 - Massa fresca total e comercial de alface-americana, cv. Raider, em função de doses de sulfato de zinco. Três Pontas - MG, UFLA, 2002. 
TABELA 1 - Massa fresca total e comercial $\left(\mathrm{g}_{\text {planta }}{ }^{-1}\right)$ de alface-americana, cv. Raider, em função da época de aplicação de sulfato de zinco. Três Pontas - MG, UFLA, 2002.

\begin{tabular}{cccc}
\hline \multirow{2}{*}{ Características } & \multicolumn{3}{c}{ Épocas de aplicação (dias após transplante) } \\
\cline { 2 - 4 } & $\mathbf{1 4}$ & $\mathbf{2 1}$ & $\mathbf{2 8}$ \\
\hline Massa fresca total & $988 \mathrm{a}$ & $941 \mathrm{~b}$ & $965 \mathrm{ab}$ \\
Massa fresca comercial & $593 \mathrm{a}$ & $557 \mathrm{~b}$ & $567 \mathrm{ab}$ \\
\hline
\end{tabular}

${ }^{1}$ Médias seguidas pela mesma letra nas linhas, não diferem entre si, pelo teste de Tukey ao nível de 5\% de probabilidade.

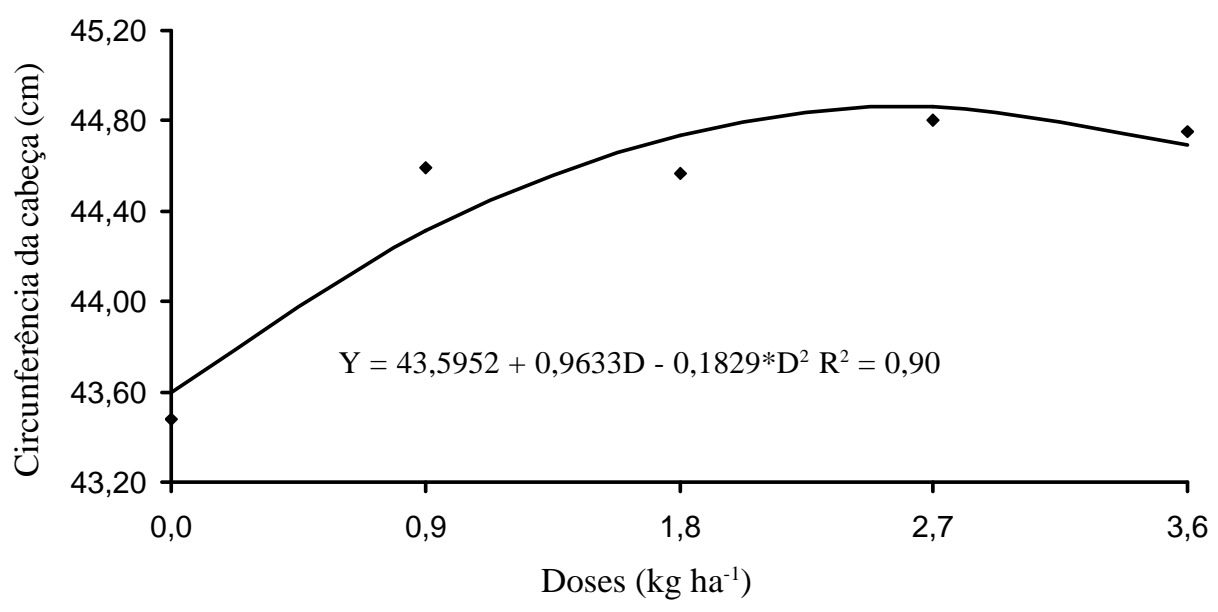

FIGURA 2 - Circunferência da cabeça comercial de alface-americana, cv. Raider, em função das doses de sulfato de zinco. Três Pontas - MG, UFLA, 2002.

\section{CONCLUSÃO}

Diante desses resultados, para as condições e época em que foi conduzido, conclui-se que para a cultura da alface-americana, a aplicação de $2,63 \mathrm{~kg} \mathrm{ha}^{-1}$ de sulfato de zinco aos 14 dias após o transplante proporciona maior rendimento produtivo.

\section{REFERÊNCIAS BIBLIOGRÁFICAS}

CAKMAK, I.; MARSCHNER, H. Increase in membrane permeability and exudation in roots of zinc deficient plants. Journal of Plant Physiology, Jena, v. 132, n. 3, p. 356 -361, 1988.

CHESWORTH, W. Geochemistry of micronutrients. In: MORTVEDT, J. J. Micronutrients in Agriculture. 2. ed. Madison: Soil Science Society American, 1991. p. 1-30.
COMPANHIA DE ENTREPOSTOS E ARMAZÉNS GERAIS DE SÃO PAULO - GEAGESP. Conjuntural de produtos por agência. São Paulo, 2001. Paginação irregular.

EMPRESA BRASILEIRA DE PESQUISA AGROPECUÁRIA. Sistema brasileiro de classificação de solos. Brasília, DF: Embrapa Produção de Informações, 1999. $412 \mathrm{p}$.

FAQUIN, V. Nutrição mineral de plantas. Lavras: FAEPE, 1994. $227 \mathrm{p}$.

FONTES, R. R.; LIMA, J. A.; TORRES, A. C.; CARRIJO, O. A. Efeito da aplicação de $\mathrm{Mg}, \mathrm{B}, \mathrm{Zn}$ e Mo na produção de alface. Pesquisa Agropecuária Brasileira, Brasília, v. 17, n. 2, p. 171-175, fev. 1982. 
GOMES, L.A.A.; SILVA, E. C.; FAQUIN, V. Recomendações de adubação para cultivos em ambiente protegido. In: RIBEIRO, A. C.; GUIMARÃES, P. T.; ALVAREZ, V. H. (Eds.). Recomendações para o uso de corretivos e fertilizantes em Minas Gerais: 5 aproximação. Viçosa: CFSEMG, 1999. p. $99-110$.

GREWAL, H. S.; ZHONGGU, L.; GRANHAN, R. D. Influence of subsoil zinc on dry matter production, seed yield and distribution of zinc in oilseed rape genotypes differing in zinc efficiency. Plant and Soil, Dordrecht, v. 192, n. 2, p. 181-189, 1997.

INSTITUTO BRASILEIRO DE GEOGRAFIA E ESTATISTICA. Organização do território: vilas e cidades. Disponível em: 〈http://www.Ibge.gov.brì. Acesso em: 19 set. 2002.

IORIO, A. F.; GORGOSCHIDE, L.; RENDINA, A.; BARROS, M. J. Effect of phosphorus, copper, and zinc addition on the phosphorus/copper and phosphorus/zinc interaction in lettuce. Journal Plant Nutrition, New York, v. 19, n. 3/4, p. 481-491, 1996.

KALYANARAMAN, S. B.; SIVAGURUNATHAN, P. Effects of cadmium, copper and zinc on the growth of blackgram. Journal Plant Nutrition, New York, v. 16, n. 10, p. 2029-2042, 1993.

MOREIRA, M. M.; FONTES, P. C. R.; CAMARGOS, M. I. Interação entre zinco e fósforo em solução nutritiva influenciando o crescimento e a produtividade da alface. Pesquisa Agropecuária Brasileira, Brasília, v. 36, n. 6, p. 903-909, jun. 2001.

PEASLEE, D. E.; ISARANGKURA, R.; LEGGETT, J. E. Acumulation and translocation of zinc by two cultivars. Agronomy Journal, Madison, v. 73, n. 4, p. 729-732, 1981.

SANTOS, R. H.; SILVA, F.; CASALI, V. W. D.; CONDE, A. R. Efeito residual da adubação com composto orgânico sobre o crescimento e produção de alface. Pesquisa Agropecuária Brasileira, Brasília, v. 36, n. 11, p. 13951398, nov. 2001.

WELCH, R. M.; NORVELL, W. A. Growth and nutrient uptake by barley (Hordeum vulgare L. cv. Herta): studies using an N-(2-Hydroxyethyl ethylenedinitrilotriacetic) acid-buffered nutrient solution technique: II. role of zinc in the uptake and root Ieakage of mineral nutrients. Plant Physiology, Rockville, v. 101, n. 2, p. 627-631, 1993.

YURI, J. E.; SOUZA, R. J. de; FREITAS, S. A. C. de; RODRIGUES JÚNIOR, J. C.; MOTA, J. H. Comportamento de cultivares de alface tipo americana em Boa Esperança. Horticultura Brasileira, Brasília, v. 20, n. 2, p. 229-232, jun. 2002.

ZINC, F. W. The response of head lettuce to soil application of zinc. Proceedings Society Horticultural Science, Saint Joseph, v. 69, p. 406-414, 1966. 